\title{
Efficacy and safety of PERIOdontal treatment versus usual care for Nonalcoholic liver disease: protocol of the PERION multicenter, two-arm, open-label, randomized trial
}

Yohei Kamata ${ }^{1 \dagger}$, Takaomi Kessoku² ${ }^{2 \dagger}$, Tomoko Shimizu $^{1 \dagger}$, Takashi Kobayashi $^{2}$, Takeo Kurihashi ${ }^{3}$, Satsuki Sato ${ }^{1}$, Syotaro Kuraji ${ }^{1}$, Norio Aoyama ${ }^{4}$, Tomoyuki Iwasaki ${ }^{5}$, Shogo Takashiba ${ }^{6}$, Nobushiro Hamada ${ }^{7}$, Toshiro Kodama ${ }^{8}$, Toshiyuki Tamura ${ }^{1}$, Satoshi Ino ${ }^{9}$, Takuma Higurashi ${ }^{2}$, Masataka Taguri ${ }^{10}$, Takeharu Yamanaka ${ }^{10}$, Masato Yoneda ${ }^{2}$, Haruki Usuda ${ }^{11}$, Koichiro Wada ${ }^{11}$, Atsushi Nakajima ${ }^{2}$ and Masato Minabe ${ }^{4^{*}}$

\begin{abstract}
Background: We report the first protocol for a multicenter, randomized comparison study to compare the efficacies of periodontal scaling and root-planing treatment against that of tooth-brushing treatment for nonalcoholic fatty liver disease (NAFLD) (PERION: PERIOdontal treatment for NAFLD). Nonalcoholic steatohepatitis (NASH) is an advanced form of NAFLD, which can progress to cirrhosis and hepatocellular carcinoma. Increased endotoxemia is associated with the progression of NAFLD. Periodontal bacteria possess endotoxins; Porphyromonas gingivalis is well-known as a major pathogenic bacterium in periodontitis, and serum antibody levels for $P$. gingivalis are high in patients with periodontitis. Several reports have indicated that $P$. gingivalis is related to NAFLD. This study aims to investigate the effect of periodontal treatment for liver damage, $P$. gingivalis infection, and endotoxemia on patients with NAFLD.

Methods: We will include adult patients (20-85 years old) with NAFLD, alanine aminotransferase (ALT) $\geq 40 \mathrm{IU} / \mathrm{L}$, and equivalent steatosis grade $\geq 1$ (target sample size, $n=40$ patients; planned number of patients with outcome data, $n=32$ ). Participants will be randomly assigned to one of two groups: a scaling and root-planing group or tooth-brushing as the usual group. The primary outcome will be the change in ALT levels from baseline to 12 weeks; the key secondary outcome will be the change in the serum immunoglobulin $G(\operatorname{lgG})$ antibody titer for $P$. gingivalis at 12 weeks.

(Continued on next page)
\end{abstract}

\footnotetext{
* Correspondence: minabe-m@wk9.so-net.ne.jp

${ }^{\dagger}$ Yohei Kamata, Takaomi Kessoku and Tomoko Shimizu contributed equally to this work.

${ }^{4}$ Division of Periodontology, Department of Oral Interdisciplinary Medicine, Graduate School of Dentistry, Kanagawa Dental University, 82 Inaoka-cho, Yokosuka, Kanagawa 238-8580, Japan

Full list of author information is available at the end of the article
}

C C The Author(s). 2020 Open Access This article is licensed under a Creative Commons Attribution 4.0 International License, which permits use, sharing, adaptation, distribution and reproduction in any medium or format, as long as you give appropriate credit to the original author(s) and the source, provide a link to the Creative Commons licence, and indicate if changes were made. The images or other third party material in this article are included in the article's Creative Commons licence, unless indicated otherwise in a credit line to the material. If material is not included in the article's Creative Commons licence and your intended use is not permitted by statutory regulation or exceeds the permitted use, you will need to obtain permission directly from the copyright holder. To view a copy of this licence, visit http://creativecommons.org/licenses/by/4.0/. The Creative Commons Public Domain Dedication waiver (http://creativecommons.org/publicdomain/zero/1.0/) applies to the data made available in this article, unless otherwise stated in a credit line to the data. 
(Continued from previous page)

Discussion: This study should determine whether periodontal treatment decreases liver damage, $P$. gingivalis infection, and endotoxemia in patients with NAFLD.

Trial registration: University Hospital Medical Information Network (UMIN) Clinical Trials Registry, ID: UMIN000022079.

Keywords: NAFLD, Porphyromonas gingivalis, Periodontal treatment, Lipopolysaccharides, Alanine aminotransferase, Immunoglobulin G

\section{Background}

The broad spectrum of fatty liver diseases in individuals who consume little-to-no alcohol is called nonalcoholic fatty liver disease (NAFLD) and includes nonalcoholic steatohepatitis (NASH). NASH is an increasingly common cause of chronic liver disease worldwide and is associated with increased liver-related mortality and hepatocellular carcinoma [1-3]. NASH progresses to cirrhosis in $15-20 \%$ of the affected individuals and is a rising indication for liver transplantation [4]. However, approved therapies for NASH have not yet been established; therefore, preventive therapies to inhibit the progression of fatty liver disease to NASH are required.

Periodontal disease is an infectious disease of the gums and tissues surrounding the teeth and causes tooth loss resulting from the destruction of tooth-supporting tissues. The incidence rate of periodontitis is $>47 \%$ in adults in the USA [5]. More than 700 bacterial species or phylotypes have been detected in the oral cavity [6]. Some species/ complexes are closely associated with advanced periodontal lesions, such as Porphyromonas gingivalis, Treponema denticola, Tannerella forsythia, Prevotella intermedia, Fusobacterium nucleatum, and Aggregatibacter actinomycetemcomitans [7, 8]. Among them, $P$. gingivalis, a gram-negative anerobic bacterium, is the major etiologic agent that contributes to periodontal disease progression and bone and tissue destruction $[9,10]$. The lipopolysaccharide (LPS) cell-wall component of $P$. gingivalis is one of the virulence factors that trigger a wide range of host responses, including the production of proinflammatory cytokines, anti-inflammatory cytokines, and chemokines [11]. These cytokines and inflammatory mediators play important roles in the progression of periodontitis at the stage where host immune and inflammatory responses lead to the destruction of periodontal tissue under the influence of multiple behavioral, environmental, and genetic factors [12].

Recently, several studies have reported the relationship between NAFLD and periodontal disease [13, 14]. Yoneda et al. [15] reported that the detection frequency of $P$. gingivalis in the saliva of patients with NAFLD and patients with NASH was significantly higher than that in nonNAFLD control subjects. Moreover, they presented preliminary evidence to suggest that nonsurgical periodontal treatments in 10 patients with NAFLD for 3 months ameliorated the liver function parameters, such as the serum levels of aspartate aminotransferase (AST) and alanine aminotransferase (ALT). Consequently, it is thought that infection with a periodontal pathogen, mainly $P$. gingivalis, is associated with fibrosis severity in patients with NAFLD and that the prevention and elimination of $P$. gingivalis infection by periodontal treatment may have a beneficial effect on the management of NASH (Fig. 1).

Therefore, we hypothesized that the elimination of oral infection, including $P$. gingivalis infection, by periodontal treatment in patients with NAFLD would ameliorate NAFLD-related clinical markers. We performed a clinical study to confirm the preliminary finding under collaborative medical and dental care.

Thus, we have devised a prospective, multicenter, randomized comparison trial to evaluate periodontal treatment as a candidate for NAFLD treatment. This is the first protocol for a randomized comparison trial for periodontal treatment against NAFLD in humans.

\section{Methods \\ Design}

The PERION trial is designed as a prospective, multicenter, two-arm, randomized comparison study to test the efficacy of the 12-week scaling and root-planing group versus the tooth-brushing group in NAFLD with moderate periodontitis. The study will recruit 40 adults and evaluate the efficacy and safety of periodontal treatment for 60 weeks, with the primary endpoint at 12 weeks. The study design is shown in Fig. 2.

\section{Recruitment process and allocation}

The PERION trial patient population will be derived from the Kanagawa Dental University Yokohama Clinic, Kanagawa Dental University, Iwasaki Internal Medicine Clinic, and the Yokohama City University Hospital Cohort. The randomized allocation will be conducted at Yokohama City University. Eligible patients will be screened by the principal and sub-investigator (gastroenterologists and periodontists). Patient recruitment will be performed $8 \mathrm{~h}$ a day, 5 days a week. 


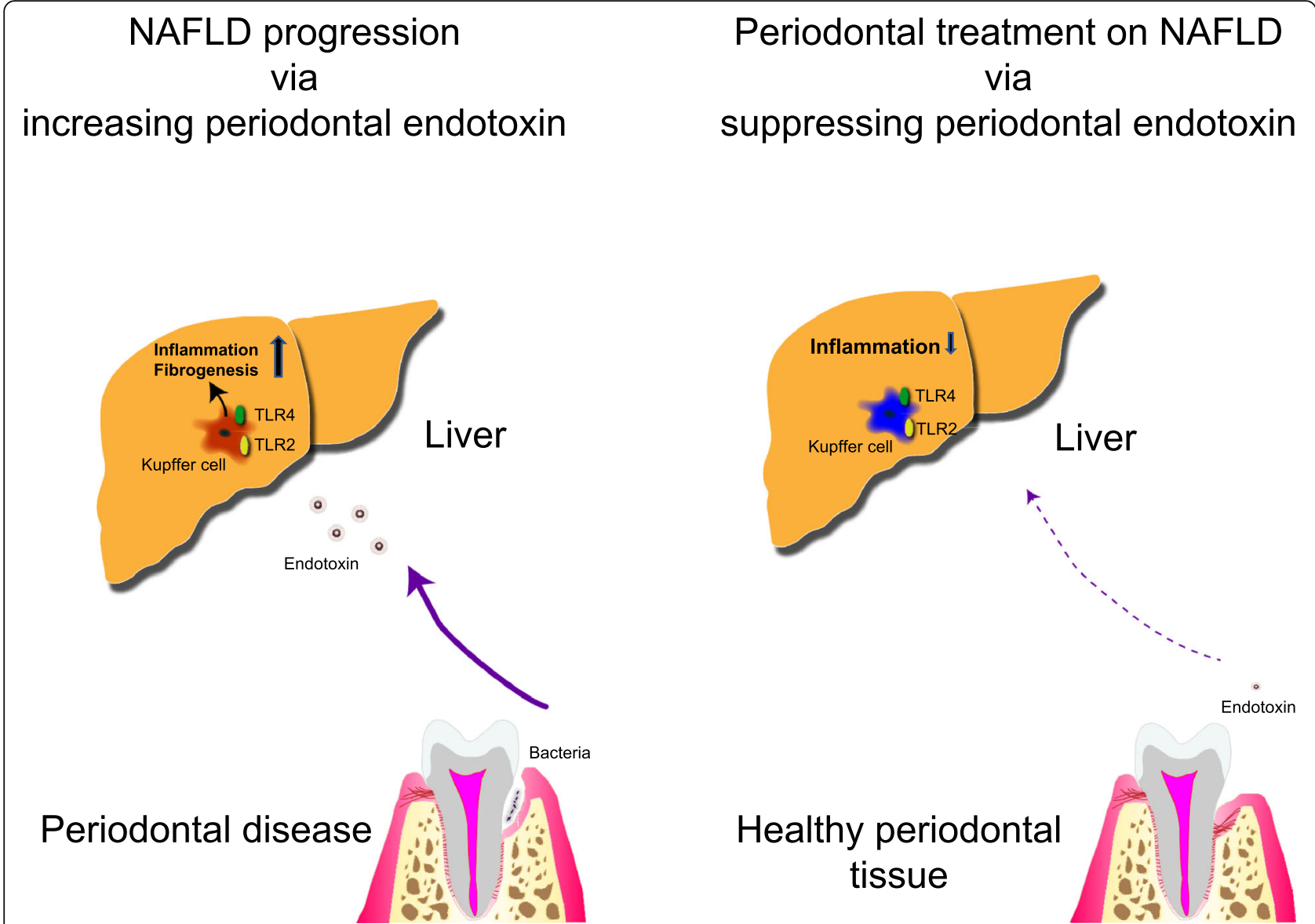

Fig. 1 Schematic overview of periodontal treatment of NAFLD suppressing periodontal endotoxin. NAFLD nonalcoholic fatty liver disease, TLR2/ TLR4 toll-like receptors 2 and 4

\section{Endpoint detection}

In the short-term studies (Phases I and IIa), aimed primarily at detecting wasted signals to make direct decisions on further development, a sustained improvement in AST and ALT levels will be useful as the endpoint of PERION (Fig. 3) [16]. While the use of ALT as a surrogate marker for NAFLD is controversial, studies have shown that ALT reduction is associated with reduced hepatocyte damage and liver inflammation [17], but not steatosis [18]. Because there are no other commonly established noninvasive biomarkers for use in the NAFLD/NASH clinical trial, ALT reduction was selected as the primary endpoint of this trial. To assist the primary endpoint, several secondary endpoints were selected to assess the pathogenesis of NAFLD using noninvasive methods (Fig. 3). Therefore, the primary endpoint of PERION is set as a change in ALT levels from baseline after 12 weeks of intervention. Also, the PERION study will evaluate periodontal treatment as a candidate for the first treatment to improve NAFLD pathogenesis via decreasing $P$. gingivalis count. Therefore, the key secondary endpoint is the serum immunoglobulin G (IgG) antibody titer of P. gingivalis [19].

\section{Periodontal disease examination}

The subject will be examined to assess the amount of periodontal disease bacteria ( $P$. gingivalis, etc.), degree of infection, and periodontal disease severity. All examinations will be performed by two periodontal disease specialists enrolled at Kanagawa Dental University Hospital and Kanagawa Dental University Yokohama Clinic. The amount of $P$. gingivalis in saliva is measured by quantitative polymerase chain reaction (qPCR), and the infection level of periodontal disease is examined using a serum IgG antibody titer test for $P$. gingivalis FDC381 by enzyme-linked immunosorbent assay (ELISA). The severity of periodontal disease will be examined by probing depths, clinical attachment levels, gingival bleedings on probing (BOP) at six sites per tooth using a calibrated periodontal probe, and the stability of the teeth.

\section{Periodontal treatment}

The primary purpose of nonsurgical periodontal treatment is to control periodontal infection of 

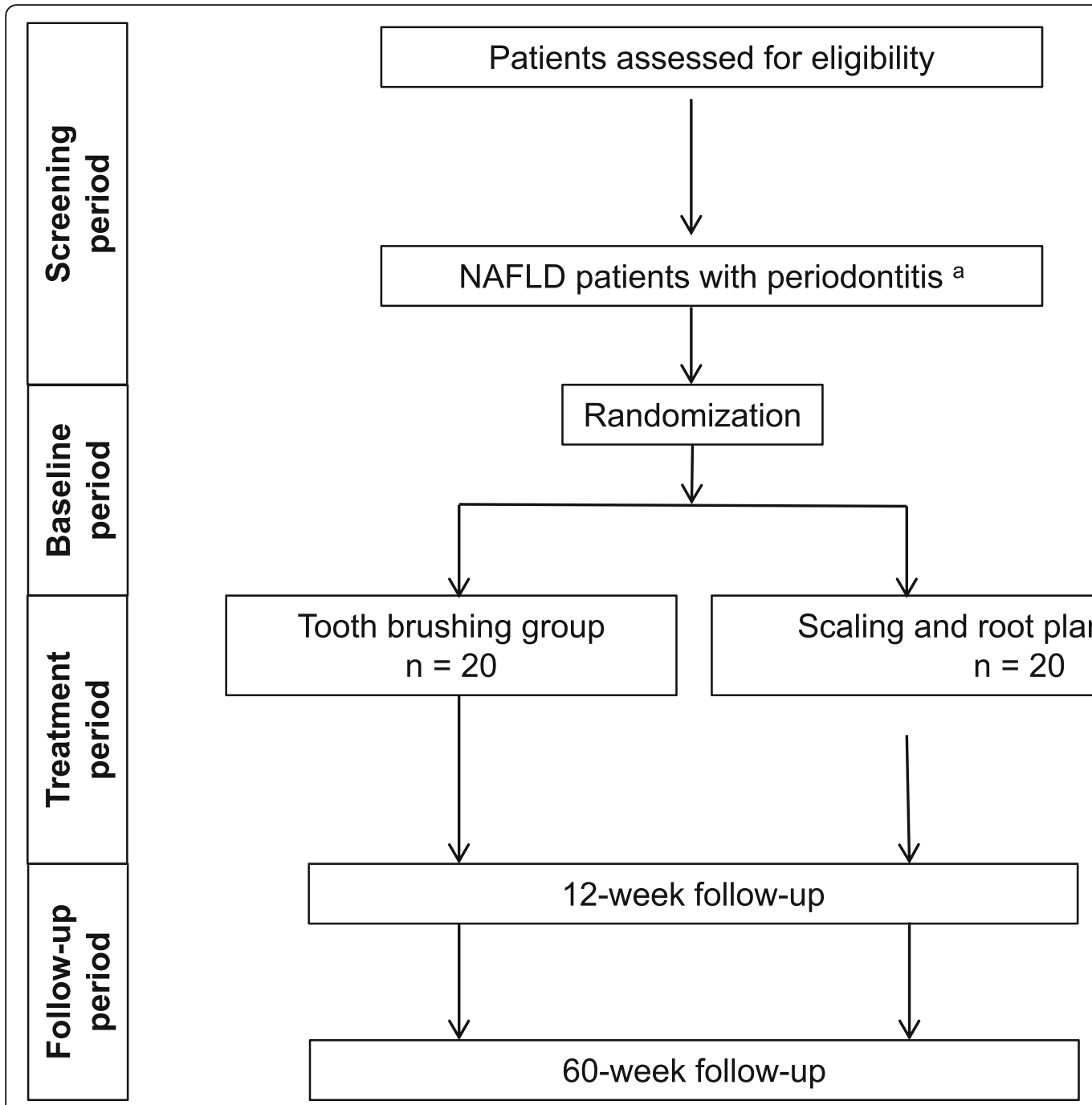

Fig. 2 Study design for PERION. Planned sample size, $N=32$; enrolled, $N=40$. PERION, PERIOdontal treatment for NAFLD, NAFLD nonalcoholic fatty liver disease

microorganisms by removing bacterial biofilm, calculus, and toxins from the root surface. According to a review of the scientific literature, mechanical nonsurgical periodontal treatment significantly reduces the inflammation level and the probing pocket depth and increases the clinical attachment level [20]. The successful treatment of plaque-induced periodontitis will recover periodontal health, but gingival recession often occurs. At first, instruction on correct brushing of the teeth will be given to patients with periodontal disease (tooth-brushing group). Then, the removal of supra- and sub-gingival bacterial plaque/ biofilm and calculus by periodontal scaling and rootplaning will be performed. Quadrant/sextant-wise instrumentation (conventional staged debridement, CSD) will be performed (scaling and root-planing group).

\section{NAFLD treatment}

We will provide standard lifestyle modification recommendations that each site can provide to the patients at the time that the informed consent document is signed. We will recommend a hypocaloric diet (daily calorie reduction of $500-1000 \mathrm{kcal}$ ) with reduced consumption of processed carbohydrates and fructose-containing beverages and will recommend the performance of moderateintensity exercise for $30-45 \mathrm{~min}$ three to four times/ week. This diet and exercise therapy will be performed without changing the prescription.

\section{Sample size determination}

Our previous pilot study showed that nonsurgical periodontal treatments on 10 NAFLD patients for 3 months ameliorated the liver function parameters, such as the serum levels of AST and ALT [15]. It showed a mean 
Primary efficacy endpoint

Changes in ALT level from baseline to 12 weeks

\section{Key secondary efficacy endpoint}

Changes in serum $\lg G$ antibody titer for $P$. gingivalis from baseline to 12 weeks

\section{Other secondary efficacy endpoints}

Changes in liver fat content (CAP and/or PDFF) from baseline to 12 weeks

Changes in liver stiffness (VCTE and/or MRE) from baseline to 12 weeks

Changes in EAA from baseline to 12 weeks

Changes in oral bacteria from baseline to 12 weeks

Changes in liver function biomarker (AST, GGT, ALP, and T-Bil) level from baseline to 12 weeks

Changes in inflammatory biomarker (ferritin, CK-18, TNF- $\alpha$, IL-6, and h-CRP) level from baseline to 12 weeks

Changes in fibrotic biomarker (type IV collagen 7S, and M2BP) level from baseline to 12 weeks

Changes in diabetic biomarker (blood glucose, insulin, and HOMA-IR) level from baseline to 12 weeks

Changes in lipid biomarker (T-Cho, LDL-C, TG, and HDL-C) level from baseline to 12 weeks

Fig. 3 Efficacy endpoints for PERION. ALP alkaline phosphatase, ALT alanine aminotransferase, AST aspartate aminotransferase, BMI body mass index, CAP controlled attenuation parameter, CK-18 cytokeratin 18, CRP C-reactive protein, EAA endotoxin activity assay, GGT Y-glutamyltransferase, h-CRP high-sensitivity C-reactive protein, HDL-C high-density lipoprotein-cholesterol, HOMA-IR homeostasis model assessment of insulin resistance, $H R Q O L$ health-related quality of life, IL-6 interleukin-6, LDL-C low-density lipoprotein-cholesterol, M2BP Mac-2 binding protein, MRE magnetic resonance elastography, NAFLD nonalcoholic fatty liver disease, PERION PERIOdontal treatment for NAFLD, PDFF proton density fat fraction, SF-8 short form-8, QOL quality of life, T-Bil total bilirubin, T-Cho total cholesterol, TG triglycerides, TNF-a tumor necrosis factor-a, VCTE vibrationcontrolled transient elastography

ALT change of -25.0 (IU/L), with a standard deviation of 25 . On the basis of these data, the sample size is determined to guarantee the power of the analysis of variance $F$ test. Assuming the mean changes in ALT in the no-treatment group and active treatment groups to be 0 and -25 , respectively, with a common standard deviation of 25 , the required number of patients per group with a power of $80 \%$ and a two-sided significance level of $5 \%$ was calculated to be 16 . We aim to recruit a total of 40 patients (scaling and root- planing treatment, 20, tooth-brushing treatment, 20) to compensate for the dropout patients.

\section{Eligibility criteria}

Patients with NAFLD who will be recruited in this study must satisfy the inclusion and exclusion criteria presented in Tables 1 and 2. Fatty liver, steatosis grade and fibrosis stage will be assessed using noninvasive methods (ultrasound, vibration-controlled transient elastography (VCTE), and magnetic resonance imaging (MRI)).

Table 1 Efficacy and safety of PERIOdontal treatment versus usual care for Nonalcoholic liver disease (PERION) inclusion criteria

\begin{tabular}{ll}
\hline Criteria type & Description of inclusion criteria \\
\hline Sex and age & Men and women: $20-85$ years of age \\
Diet and exercise therapy & Patients with NAFLD who did not respond to 3-month diet and exercise therapy \\
ALT levels & Patients with an ALT level of $>40 \mathrm{IU} / \mathrm{L}$ at the start of this study \\
Fatty liver & Patients with a diagnosis of fatty liver based on abdominal ultrasonography ${ }^{\mathrm{a}}$ \\
Steatosis grade & Patients with the equivalent of steatosis grade $\geq 1$ on CAP (using FibroScan) and/or PDFF (using MRI) \\
Fibrosis stage & Patients with the equivalent of fibrosis stage $<4$ on TE (FibroScan) and/or MRE \\
Alcohol consumption & Patients with no habitual alcohol consumption (i.e., consumption of ethanol $>30 \mathrm{~g} /$ day in men and $>20 \mathrm{~g} /$ day in women) \\
Periodontitis & Patients with chronic moderate periodontitis (holding rate of periodontal pocket depth of $>4$ mm is $>10$ sites) \\
Other & Patients who can provide written consent to participate in this research in person, follow instructions during \\
& participation in this research, undergo protocol-specified physical examination and other examinations, and report \\
& symptoms or events
\end{tabular}

ALT alanine aminotransferase, CAP controlled attenuation parameter, MRE magnetic resonance elastography, MRI magnetic resonance imaging, NAFLD nonalcoholic fatty liver disease, PERION PERIOdontal treatment for NAFLD, PDFF proton density fat fraction, VCTE vibration-controlled transient elastography. ${ }^{a}$ Criteria of fatty liver, as defined by the existence of hepatorenal echo contrast. ${ }^{b}$ Defined by CAP $\geq 236 \mathrm{~dB} / \mathrm{m}$ and/or PDFF $\geq 5.2 \%$. ${ }^{\mathrm{C} D e f i n e d}$ by VCTE $<14 \mathrm{kPa}$ and/ or MRE $<6.7 \mathrm{kPa}$ 
Table 2 Efficacy and safety of PERIOdontal treatment versus usual care for Nonalcoholic liver disease (PERION) exclusion criteria

\begin{tabular}{ll}
\hline Criteria type & Description of exclusion criteria \\
\hline Liver comorbidity & Patients with any other concurrent liver disease, such as hepatitis C, hepatitis B, or autoimmune hepatitis \\
& Patients with drug-induced symptomatic NAFLD \\
Other comorbidity & Patients with concurrent or past history of any serious cardiac, vascular, hematological, respiratory, hepatic, \\
& renal, gastrointestinal, or neuropsychiatric disease \\
& Patients with a history of abdominal or gastrointestinal surgery, except appendicitis \\
Medication & Patients with any change to their orally administered medications within 3 months before informed consent \\
& Patients with diabetes mellitus being treated with insulin injections \\
Other & Patients who have participated in any other clinical study and received study treatment within 1 month before \\
& the start of this research (counted from the first day of study medication) \\
& Breastfeeding women or women with possible pregnancy \\
& Other patients who are inappropriate as participants in this research in the opinion of the principal investigator, etc.
\end{tabular}

NAFLD nonalcoholic fatty liver disease, PERION PERIOdontal treatment for NAFLD

\section{Randomization}

Before providing informed consent, patients will undergo a screening test as a first step to determine whether they meet the inclusion criteria for the study and none of the exclusion criteria. For patients determined to be eligible, the principal investigator or coinvestigator will complete a Patient Enrollment Form with the necessary information, which is sent by fax or e-mail to the Patient Enrollment Center (Yokohama City University). The Patient Enrollment Center will then confirm the eligibility of the patient based on the enrollment form, enroll and randomize the patient, and notify the principal investigator or co-investigator of the $\mathrm{Pa}$ tient ID number and the allocation number via fax or email. After an eligibility check, patients will be randomly assigned to receive periodontal treatment or no treatment at the Central Registration Center by a computer program, using a stratified block randomization method, adjusting for age ( $\geq 65 /<65$ years) and sex (male/female). Therefore, the patient assignment is concealed from the investigator.

\section{Study procedures}

The schedule of assessments for the study procedures is summarized in Table 3.

\section{Observation of adverse events}

All adverse events (AEs) during the study will be recorded with regard to the following: date of onset and date of completion (if applicable), severity of AEs, investigator's view on the relationship to periodontal treatment, information on actions taken, information on the treatment of the AE, the cause of the event (if known), and the solution or outcome. AEs classified as serious will be recorded in the serious adverse event reporting tool and reported to the sponsor. AE intensities are graded according to the National Cancer Institute's Adverse Event Common Terminology (NCI CTCAE) version 4.03, including the AE-intensity classifications shown in Table 4.

\section{Additional study procedures}

Patients must be fasted for at least $8 \mathrm{~h}$ prior to the visit. The study visits will be at $0,4,8,12$, and 60 weeks.

\section{Criteria for discontinuation of study treatment}

The study must be discontinued under the following conditions: drug-induced liver injury, unacceptable toxicity, acute viral hepatitis $\mathrm{B}$ and $\mathrm{C}$, autoimmune or alcoholic hepatitis, hypoxic or ischemic liver injury, biliary tract disease, or pregnancy.

\section{Outcome measures}

\section{Evaluation of efficacy}

The efficacy endpoints for this study are shown in Fig. 3.

\section{Evaluation of safety}

The safety and tolerability of periodontal treatment will be evaluated over the 60 weeks of treatment in NAFLD patients with periodontitis. This will include the evaluation of AEs, clinical laboratory tests, physical examination, and vital signs. The clinical laboratory tests will include liver and fasting metabolic parameters. Liver parameters will include alkaline phosphatase (ALP), AST, ALT, total bilirubin (T-Bil), and $\gamma$-glutamyltransferase (GGT).

\section{Statistical analyses}

The Full Analysis Set (FAS) will be the primary analysis set for efficacy. We define FAS to include any subjects who receive any amount of the study medication without a lack of information on the primary endpoint (complete case analysis). For the blood liver function test using ALT, the summary statistics (mean, standard deviation) will be calculated at baseline and 12 weeks. Details of the statistical design will be described in the Statistical Analysis Plan. In this study, statistical analysis will be 
Table 3 Objectives and procedures of the Efficacy and safety of PERIOdontal treatment versus usual care for Nonalcoholic liver disease (PERION) study

\begin{tabular}{|c|c|c|c|c|c|c|}
\hline \multirow[b]{3}{*}{ Study objectives } & \multicolumn{6}{|c|}{ Study time point (weeks) } \\
\hline & \multirow{2}{*}{$\begin{array}{l}\text { Screening period } \\
-4\end{array}$} & \multicolumn{4}{|c|}{ Treatment period } & \multirow{2}{*}{$\begin{array}{l}\text { Follow-up period } \\
60\end{array}$} \\
\hline & & 0 & 4 & 8 & 12 & \\
\hline \multicolumn{7}{|l|}{ Primary objective } \\
\hline Change in ALT levels from baseline & $\circ$ & $\circ$ & & & $\circ$ & $\circ$ \\
\hline \multicolumn{7}{|l|}{ Key secondary objective } \\
\hline Change in serum IgG antibody titer for $P$. gingivalis & $\circ$ & & & & $\circ$ & $\circ$ \\
\hline \multicolumn{7}{|l|}{ Other secondary objectives } \\
\hline Change in blood endotoxin activity by EAA & $\circ$ & $\circ$ & $\circ$ & $\circ$ & $\circ$ & $\circ$ \\
\hline Change in liver fat content using CAP and MRI-PDFF & $\circ$ & $\circ$ & & & $\circ$ & \\
\hline Change in liver stiffness using VCTE and MRE & $\circ$ & $\circ$ & & & $\circ$ & \\
\hline Change in oral bacterial counts using NGS and qPCR & $\circ$ & $\circ$ & & & $\circ$ & \\
\hline Change in blood parameters for liver function (AST, GTT, ALP, and T-Bil) & $\circ$ & $\circ$ & $\circ$ & $\circ$ & $\circ$ & $\circ$ \\
\hline Change in blood lipid parameters (T-Cho, LDL-C, TG, and HDL-C) & $\circ$ & $\circ$ & $\circ$ & ○ & $\circ$ & $\circ$ \\
\hline Change in blood parameters related with inflammation in NAFLD (ferritin, CK-18, TNF-a, IL & L-6, and $h-C R P)$ & $\circ$ & & & $\circ$ & \\
\hline Change in blood parameters related with fibrotic marker in NAFLD (type IV collagen 7S) & & $\circ$ & & & $\circ$ & \\
\hline Change in blood diabetic factors (blood glucose, insulin, and HOMA-IR) & & $\circ$ & $\circ$ & $\circ$ & $\circ$ & $\circ$ \\
\hline Change in BMl & $\circ$ & $\circ$ & $\circ$ & $\circ$ & $\circ$ & $\circ$ \\
\hline Assessment of periodontal treatment safety & & $\circ$ & $\circ$ & $\circ$ & $\circ$ & $\circ$ \\
\hline Dropout ratio in each group & & $\circ$ & $\circ$ & $\circ$ & $\circ$ & $\circ$ \\
\hline \multicolumn{7}{|l|}{ Tertiary objectives } \\
\hline Change in blood parameters for renal function (BUN, Cr, eGFR) & $\circ$ & $\circ$ & $\circ$ & $\circ$ & $\circ$ & $\circ$ \\
\hline Change in HRQOL using SF-8 ${ }^{\mathrm{TM}}$ & $\circ$ & $\circ$ & & & $\circ$ & $\circ$ \\
\hline
\end{tabular}

All objectives will be compared between the periodontal scaling and root-planing treatment group and the tooth-brushing treatment group. ALP alkaline phosphatase, ALT alanine transaminase, AST aspartate transaminase, BMI body mass index, BUN blood urea nitrogen, CAP controlled attenuation parameter, CK-18 cytokeratin $18, \mathrm{Cr}$ creatinine, EAA endotoxin activity assay, eGFR estimated glomerular filtration rate, FBS fasting blood sugar, GGT $\gamma$-glutamyltransferase, $h$-CRP high-sensitivity C-reactive protein, HDL-C high-density lipoprotein-cholesterol, HOMA-IR homeostasis model assessment of insulin resistance, $H R Q O L$ health-related quality of life, IL-6 interleukin-6, LDL-C low-density lipoprotein-cholesterol, MRE magnetic resonance elastography, MRI magnetic resonance imaging, NAFLD nonalcoholic fatty liver disease, NGS next-generation sequencer; PERION, PERIOdontal treatment for NAFLD, PDFF proton density fat fraction, $q P C R$ quantitative polymerase chain reaction, SF-8 short form-8, T-Bil total bilirubin, T-Cho total cholesterol, TG triglycerides, TNF- $a$ tumor necrosis factor-a

performed mainly for the following items. For the primary analysis, (ALT after treatment - ALT at baseline) will be sought for each subject and the corresponding Wilcoxon test will be performed. The significance level will be $5 \%$ on both sides. In addition to $p$ values, we will provide point estimates with $95 \%$ confidence intervals. As a sensitivity analysis, the two groups would be compared using the analysis of covariance using the primary endpoint as an outcome, adjusting for ALT at baseline as a covariate. For the key secondary endpoint, $P$. gingivalis IgG antibody titer in the blood (values after treatment - values at baseline) will be sought for each subject and the paired Wilcoxon test will be performed. Subgroup analysis will be conducted. Stratified analysis will be performed using $P$. gingivalis bacterial content, proton density fat fraction (PDFF), magnetic resonance

Table 4 Classifications of adverse event (AE) intensity

\begin{tabular}{ll}
\hline Grade & Description \\
\hline Grade 1 (mild) & Asymptomatic or mild symptoms; clinical or diagnostic observations only; intervention not indicated \\
Grade 2 (moderate) & Minimal, local, or noninvasive intervention indicated; limiting age-appropriate instrumental ADL \\
Grade 3 (severe) & $\begin{array}{l}\text { Medically significant but not immediately life-threatening; hospitalization or prolongation of hospitalization i } \\
\text { ndicated; disabling; limiting self-care ADL }\end{array}$ \\
Grade 4 (life-threatening) & Life-threatening consequences; urgent intervention indicated \\
Grade 5 (death) & Death related to AE
\end{tabular}


elastography (MRE), and periodontal pocket as indices. Stratified analysis will be performed using above/below the median of $P$. gingivalis bacterial content, endotoxin, PDFF, MRE, and periodontal pocket depth. We hypotheses a larger reduction in ALT with the intervention in the patient group with high $P$. gingivalis bacterial content, high endotoxin levels, high PDFF, low MRE, and deep periodontal pockets at baseline. Therefore, we conducted multivariable linear regression with difference in ALT between groups as dependent variable and at least the treatment group, the respective subgroup variable (categorized), and the interaction term (treatment group $\times$ subgroup variable) as independent variables. The significance level will be $5 \%$ on both sides. In addition to p values, we will provide point estimates with $95 \%$ confidence intervals. As a sensitivity analysis, the two groups will be compared using analysis of covariance using the key secondary endpoint as an outcome adjusting for $P$. gingivalis IgG antibody titer at baseline as a covariate. As the safety analysis, the incidence and severity of AEs and reactions will be calculated.

Trial Steering Committee and Data Monitoring Committee The Trial Operation Committee is integrated and consists of three persons appointed by independent clinical and basic investigators (a general internist in primary care, a palliative care specialist and a statistician from Yokohama City University School of Medicine). They provide overall supervision and ensure that all registered trials. These investigators are anonymous and randomly selected. The Independent Data Monitoring Committee will be established with two persons from the Department of Biostatistics, Yokohama City University School of Medicine. The management team will observe progress and data monthly by phone call, mail, and/or webconferencing. If the Monitoring Committee decides that on-site monitoring is necessary, monitoring members will visit the site for face-to-face monitoring.

\section{Discussion}

This is the first study proposed to explore the effect of periodontal treatment on NAFLD patients with periodontitis. The primary endpoint used in previous studies was the liver histology, which was evaluated by a liver biopsy (Pivens, Flint, and Golden study) [21-24]. Liver histology endpoints, such as the complete resolution of $\mathrm{NASH}$, are considered surrogates for preventing cirrhosis (i.e., they are thought to predict a clinical benefit but are not direct measurements of it). However, due to increased cost, possible risk, inter- and intra-observer bias, and healthcare resource utilization, an invasive liver biopsy is poorly suited as a diagnostic test for such a prevalent condition [25]. Furthermore, the histological lesions of NASH are unevenly distributed throughout the liver parenchyma; therefore, liver biopsy sampling error can result in substantial stratification and staging inaccuracies [26]. Currently, the noninvasive methods used to assess NASH progression are not robust enough to replace liver biopsy. We considered that it is important to perform noninvasive, safe, low-cost, and shortterm clinical trials as proof-of-concept studies. Many studies reported that the steatosis grade can be evaluated using controlled attenuation parameter (CAP) [27-32], which is based on the properties of ultrasonic signals acquired by VCTE, and MRI-PDFF [33, 34], which is an MRI-based method for quantitatively assessing hepatic steatosis and is available from several manufacturers of MRI scanners. Moreover, VCTE and MRE have superior diagnostic ability to evaluate steatosis and fibrosis in patients with NAFLD $[35,36]$. Therefore, we consider that noninvasive evaluation of NAFLD pathogenesis using VCTE and MRI could replace invasive methods, such as liver biopsy, as a proof-of-concept study. Therefore, noninvasive methods, such as VCTE and MRI, to assess NASH/NAFLD progression, were included as secondary endpoints to compare against liver biopsy.

Clinical research in Japanese university students has suggested that having periodontal disease in young men was significantly associated with an increased level of ALT [37]. In addition, the incidence of periodontal disease in healthy Japanese women was reported to be significantly increased with elevated serum levels of AST, ALT, and cholinesterase [38]. Furthermore, an observational study with annual workplace health check-ups at a company in Japan reported an association between periodontal condition and the combination of elevated ALT and metabolic syndrome (MetS) in men [39]. Besides, it has been suggested that more severe periodontal disease is associated with increased serum levels of GGT, a liver biochemical parameter, in Japanese adults with no alcohol-drinking habits [40]. In an in-vivo mouse study, it was demonstrated that areas of fibrosis with proliferation of hepatic stellate cells and collagen formation were observed in mice with $P$. gingivalis infection fed on a high-fat diet. In addition, in steatotic hepatocytes, the expression of toll-like receptor 2 (TLR2), one of the $P$. gingivalis-LPS receptors, was upregulated. $P$. gingivalis-LPS further increased messenger ribonucleic acid (mRNA) levels of palmitate-induced inflammasome and proinflammatory cytokines in steatotic hepatocytes [41]. That is to say, the dental infection of $P$. gingivalis exacerbated the pathological progression of NASH from simple steatohepatitis to steatohepatitis with fibrosis through the upregulation of the $P$. gingivalis-LPS-TLR2 pathway and activation of inflammasomes. Recently, evidence in mice has shown that disturbance of the gut microbiota composition by orally derived periodontopathic bacteria, such as $P$. gingivalis, may be a 
causal mechanism linking periodontitis and systemic disease including NAFLD [42-44].

In conclusion, this study should determine whether periodontal treatment to decrease endotoxin levels and treat $P$. gingivalis infection improves the disease status of patients with NAFLD. The PERION study is the first randomized controlled study suppressing hyper-endotoxemia in NAFLD with periodontitis. This study should allow the assessment of the efficacy and safety of periodontal treatment in a larger population of NAFLD patients with periodontitis.

\section{Strengths}

To our knowledge, no direct comparison has been made between randomized controlled groups of patients with NAFLD with periodontitis. The strength of this study is that non-invasive MRI-PDFF will be used to assess changes in hepatic fat mass, as opposed to liver biopsy. Importantly, improved laboratory tests for periodontal disease and $P$. gingivalis removal may correlate with improved liver fat mass and liver function.

\section{Limitations}

There are several limitations in our study. First, the small sample size and short treatment period (3 months) limit our findings. Second, it is important to differentiate NASH in NAFLD patients; however, at present, the gold standard for such differentiation is liver-tissue diagnosis by liver biopsy. In this study, NAFLD patients would not have undergone liver biopsy. Third, our protocol is open-label. Fourth, the specified primary and key secondary outcomes are only surrogate outcomes. The extent of decrease in ALT or IgG antibody titers will translate into improved quality of life (QOL) or any other more patient-relevant outcomes of NAFLD patients will not be determined in the present trial.

\section{Trial status}

Recruitment of participants begun in August 2015 and will be open until March 2020.

Current approved protocol: Version 1.3, 24 January 2018.

\footnotetext{
Abbreviations

ALP: Alkaline phosphatase; ALT: Alanine aminotransferase; AST: Aspartate aminotransferase; BMI: Body mass index; CAP: Controlled attenuation parameter; CK-18: Cytokeratin 18; CRP: C-reactive protein; EAA: Endotoxin activity assay; eGFR: Estimated glomerular filtration rate; GGT: - $^{-}$ glutamyltransferase; h-CRP: High-sensitivity C-reactive protein; HDL-C: Highdensity lipoprotein-cholesterol; HOMA-IR: Homeostasis model assessment of insulin resistance; HRQOL: Health-related quality of life; IEC: Independent Ethics Committee; IL-6: Interleukin-6; IRB: Institutional Review Board; LDLC: Low-density lipoprotein-cholesterol; LPS: Lipopolysaccharide; LSM: Liver stiffness measurement; MRE: Magnetic resonance elastography; MRI: Magnetic resonance imaging; NAFLD: Nonalcoholic fatty liver disease; NASH: Nonalcoholic steatohepatitis; PDFF: Proton density fat fraction; QOL: Quality of life; qPCR: Quantitative polymerase chain reaction; SF-8: Short form-8; T-Bil: Total bilirubin; T-Cho: Total cholesterol; TG: Triglyceride;
}

TLR2: Toll-like receptor 2; TLR4: Toll-like receptor 4; TNF-a: Tumor necrosis factor-a; VCTE: Vibration-controlled transient elastography

\section{Acknowledgements}

We would like to express our gratitude to the patients, their families, the study coordinators, and investigators, as well as the PERION study team.

\section{Authors' contributions}

YK, T Kessoku, AN, and MM conceived the study idea. TS, NA, and T Kobayashi conducted feasibility phase work. Recruitment of participants and follow-up will be performed by T Kurihashi, SS, SK, TI, T Kodama, TT, and SI. Analysis and interpretation of data will be conducted by TH, MT, TY, and MY. $\mathrm{HU}$, and KW will perform the bioinformatics analysis. ST and $\mathrm{NH}$ will measure periodontal pathogens. All authors have read and approved the final manuscript.

\section{Funding}

This work was supported by the Ministry of Health, Labor, and Welfare, Japan (grant number 16 K11872).

\section{Availability of data and materials}

Not applicable

\section{Ethics approval and consent to participate}

The study protocol conforms to the Helsinki Declaration [45] and the Ethics Guidelines for Clinical Research notified by the Ministry of Health, Labor, and Welfare, Japan [46]. The study is projected and organized according to the Consolidated Standards of Reporting Trials 2010 guidelines [47]. This trial is registered at the University Hospital Medical Information Network (UMIN) Clinical Trials Registry as UMIN000022079. The study protocol was recognized by the Ethics Committees of Kanagawa Dental University, Kanagawa Dental University Yokohama Clinic, Iwasaki Internal Medicine Clinic, Yokohama City University Hospital, and Okayama University. The protocol and informed consent forms were accepted by the Ethics Committee at each of the participating institutions. Informed consent will be obtained from all participants prior to registration. During and after the trial, we are committed to complying with the requirements of all Institutional Review Boards/ Independent Ethics Committees.

The Ethics Committee of Kanagawa Dental University Hospital approved the study protocol on 21 August 2015. This trial is registered with the University Hospital Medical Information Network (UMIN) Clinical Trials Registry (unique ID: UMIN000022079, date of registration: 15 July, 2015). Written informed consent will be obtained from all participants before enrollment. The protocol and any information supplied to gain informed consent were approved by a qualified Institutional Review Board/Independent Ethics Committee prior to subject enrollment.

\section{Consent for publication}

Not applicable

\section{Competing interests}

The authors declare that they have no competing interests.

\section{Author details}

${ }^{1}$ Department of Highly Advanced Oral Stomatology, Yokohama Clinic, Kanagawa Dental University, 3-31-6 Tsuruya-cho, Kanagawa, Yokohama, Kanagawa 221-0835, Japan. ${ }^{2}$ Department of Gastroenterology and Hepatology, Yokohama City University Graduate School of Medicine, 3-9 Fukuura, Kanazawa-ku, Yokohama 236-0004, Japan. ${ }^{3}$ Department of Internal Medicine, Yokohama Clinic, Kanagawa Dental University, 3-31-6 Tsuruya-cho, Kanagawa, Yokohama, Kanagawa 221-0835, Japan. ${ }^{4}$ Division of

Periodontology, Department of Oral Interdisciplinary Medicine, Graduate School of Dentistry, Kanagawa Dental University, 82 Inaoka-cho, Yokosuka, Kanagawa 238-8580, Japan. ${ }^{5}$ Iwasaki Internal Medicine Clinic, 1-1-5 Furu-ruyokohama1F, Kamihoshikawa, Hodogaya-ku Yokohama, Kanagawa 240-0042, Japan. ${ }^{6}$ Department of Pathophysiology - Periodontal Science, Okayama University Graduate School of Medicine, Dentistry and Pharmaceutical Sciences, 2-5-1 Shikata-cho, Kita-ku, Okayama 700-8525, Japan. ${ }^{7}$ Division of Microbiology, Department of Oral Science Graduate School of Dentistry, Kanagawa Dental University, 82 Inaoka-cho, Yokosuka, Kanagawa 238-8580, Japan. ${ }^{8}$ Department of Implantology and 
Periodontology, Graduate School of Dentistry, Kanagawa Dental University, 3-31-6 Tsuruya-cho, Kanagawa, Yokohama, Kanagawa 221-0835, Japan. ${ }^{9}$ Division of Prosthetic Dentistry, Department of Highly Advanced Stomatology, Graduate School of Dentistry, Kanagawa Dental University, 3-31-6 Tsuruya-cho, Kanagawa, Yokohama, Kanagawa 221-0835, Japan. ${ }^{10}$ Department of Biostatistics, Yokohama City University Graduate School of Medicine, 3-9 Fukuura, Kanazawa-ku, Yokohama 236-0004, Japan.

${ }^{11}$ Department of Pharmacology, Shimane University School of Medicine, 89-1 Enya-cho Izumo, Shimane 693-0581, Japan.

\section{Received: 5 October 2019 Accepted: 24 February 2020} Published online: 23 March 2020

\section{References}

1. Page JM, Harrison SA. NASH, and HCC. Clin Liver Dis. 2009;13:631-47.

2. Jimba S, Nakagami T, Takahashi M, Wakamatsu T, Hirota Y, Iwamoto Y, et al. Prevalence of non-alcoholic fatty liver disease and its association with impaired glucose metabolism in Japanese adults. Diabet Med. 2005;22: 1141-5.

3. Sanyal A, Poklepovic A, Moyneur E, Barghout V. Population-based risk factors and resource utilization for HCC: US perspective. Curr Med Res Opin. 2010;26:2183-91.

4. Agopian VG, Kaldas FM, Hong JC, Whittaker M, Holt C, Rana A, et al. Liver transplantation for nonalcoholic steatohepatitis: the new epidemic. Ann Surg. 2012;256:624-33.

5. Eke PI, Dye BA, Wei L, Thornton-Evans GO, Genco RJ. Prevalence of periodontitis in adults in the United States: 2009 and 2010. J Dent Res. 2012;91:914-20.

6. Aas JA, Paster BJ, Stokes LN, Olsen I, Dewhirst FE. Defining the normal bacterial flora of the oral cavity. J Clin Micorbiol. 2005;43:5721-32.

7. Bodet C, Chandad F, Grenier D. Pathogenic potential of Porphyromonas gingivalis, Treponema denticola and Tannerella forsythia, the red bacterial complex associated with periodontitis. Pathol Biol (Paris). 2007;55:154-62.

8. Perez-Chaparro PJ, Gonçalves C, Figueiredo LC, Faveri M, Lobão E, Tamashiro N, et al. Newly identified pathogens associated with periodontitis: a systematic review. J Dent Res. 2014;93:846-58.

9. Holt SC, Kesavalu L, Walker S, Genco CA. Virulence factors of Porphyromonas gingivalis. Periodontol 2000. 1999;20:168-238.

10. How KY, Song KP, Chan KG. Porphyromonas gingivalis: an overview of periodontopathic pathogen below the gum line. Front Microbiol. 2016;7:53.

11. Sun Y, Li H, Yang MF, Shu W, Sun MJ, Xu Y. Effects of aging on endotoxin tolerance induced by lipopolysaccharides derived from Porphyromonas gingivalis and Escherichia coli. PLOS One. 2012;7:e39224

12. Liang S, Krauss JL, Domon H, McIntosh ML, Hosur KB, Qu H, et al. The C5a receptor impairs IL-12-dependent clearance of Porphyromonas gingivalis and is required for induction of periodontal bone loss. J Immunol. 2011;186:869-77.

13. Kuraji R, Fujita M, Ito H, Hashimoto S, Numabe Y. Effects of experimental periodontitis on the metabolic system in rats with diet-induced obesity (DIO): an analysis of serum biochemical parameters. Odontology. 2018;106: 162-70.

14. Nakahara T, Hyogo H, Ono A, Nagaoki Y, Kawaoka T, Miki D, et al. Involvement of Porphyromonas gingivalis in the progression of nonalcoholic fatty liver disease. J Gastroenterol. 2018;53:269-80.

15. Yoneda M, Naka S, Nakano K, Wada K, Endo H, Mawatari H, et al. Involvement of a periodontal pathogen, Porphyromonas gingivalis on the pathogenesis of non-alcoholic fatty liver disease. BMC Gastroenterol. 2012;12:16.

16. Park H, Shima T, Yamaguchi K, Mitsuyoshi H, Minami M, Yasui K, et al. Efficacy of long-term ezetimibe therapy in patients with nonalcoholic fatty liver disease. J Gastroenterol. 2011;46:101-7.

17. Sanyal AJ, Chalasani N, Kowdley KV, McCullough A, Diehl AM, Bass NM, et al. Pioglitazone, vitamin E, or placebo for nonalcoholic steatohepatitis. New Eng J Med. 2010;362:1675-85.

18. Suzuki A, Lymp J, St Sauver J, Angulo P, Lindor K. Values and limitations of serum aminotransferases in clinical trials of nonalcoholic steatohepatitis. Liver Int. 2006;26:1209-16.

19. Kudo C, Naruishi K, Maeda H, Abiko Y, Hino T, Iwata M, et al. Assessment of the plasma/serum IgG test to screen for periodontitis. J Dent Res. 2012;91: 1190-5.

20. American Academy of Periodontology. Comprehensive periodontal therapy: a statement by the American Academy of Periodontology*. J Periodontol. 2011;82:943-9.
21. Sanyal AJ, Brunt EM, Kleiner DE, Kowdley KV, Chalasani N, Lavine JE, et al. Endpoints and clinical trial design for nonalcoholic steatohepatitis. Hepatology. 2011:54:344-53.

22. Sanyal AJ, Friedman SL, McCullough AJ, Dimick-Santos L. Challenges and opportunities in drug and biomarker development for nonalcoholic steatohepatitis: findings and recommendations from an American Association for the Study of Liver Diseases-U.S. Food and Drug Administration Joint Workshop. Hepatology. 2015;61:1392-405.

23. Neuschwander-Tetri BA, Loomba R, Sanyal AJ, Lavine JE, Van Natta ML, Abdelmalek MF, et al. Farnesoid X nuclear receptor ligand obeticholic acid for non-cirrhotic, non-alcoholic steatohepatitis (FLINT): a multicentre, randomised, placebo-controlled trial. Lancet. 2015;385:956-65.

24. Ratziu V, Harrison SA, Francque S, Bedossa P, Lehert P, Serfaty L, et al. Elafibranor, an agonist of the peroxisome proliferator-activated receptor-a and $-\delta$, induces resolution of nonalcoholic steatohepatitis without fibrosis worsening. Gastroenterology. 2016;150:1147-59.

25. Cadranel JF. Good clinical practice guidelines for fine needle aspiration biopsy of the liver: past, present and future. Gastroenterol Clin Biol. 2002;26: 823-4.

26. Ratziu V, Charlotte F, Heurtier A, Gombert S, Giral P, Bruckert E, et al. Sampling variability of liver biopsy in nonalcoholic fatty liver disease. Gastroenterology. 2005;128:1898-906.

27. Sasso M, Beaugrand M, de Ledinghen V, Douvin C, Marcellin P, Poupon R, et al. Controlled attenuation parameter (CAP): a novel VCTE ${ }^{\mathrm{TM}}$ guided ultrasonic attenuation measurement for the evaluation of hepatic steatosis: preliminary study and validation in a cohort of patients with chronic liver disease from various causes. Ultrasound Med Biol. 2010;36:1825-35.

28. Obara N, Ueno Y, Fukushima K, Nakagome Y, Kakazu E, Kimura O, et al. Transient elastography for measurement of liver stiffness measurement can detect early significant hepatic fibrosis in Japanese patients with viral and nonviral liver diseases. J Gastroenterol. 2008;43:720-8.

29. Alisi A, Pinzani M, Nobili V. Diagnostic power of fibroscan in predicting liver fibrosis in nonalcoholic fatty liver disease. Hepatology. 2009;50:2048-9 author reply 2049-50.

30. Chang PE, Lui HF, Chau YP, Lim KH, Yap WM, Tan CK, et al. Prospective evaluation of transient elastography for the diagnosis of hepatic fibrosis in Asians: comparison with liver biopsy and aspartate transaminase platelet ratio index. Aliment Pharmacol Ther. 2008;28:51-61.

31. Chan WK, Nik Mustapha NR, Mahadeva S. Controlled attenuation parameter for the detection and quantification of hepatic steatosis in nonalcoholic fatty liver disease. J Gastroenterol Hepatol. 2014;29:1470-6.

32. de Lédinghen V, Vergniol J, Capdepont M, Chermak F, Hiriart JB, Cassinotto C, et al. Controlled attenuation parameter (CAP) for the diagnosis of steatosis: a prospective study of 5323 examinations. J Hepatol. 2014;60: 1026-31.

33. Reeder SB, Robson PM, Yu H, Shimakawa A, Hines CD, McKenzie CA, et al. Quantification of hepatic steatosis with MRI: the effects of accurate fat spectral modeling. J Magn Reson Imaging. 2009;29:1332-9.

34. Permutt Z, Le TA, Peterson MR, Seki E, Brenner DA, Sirlin C, et al. Correlation between liver histology and novel magnetic resonance imaging in adult patients with non-alcoholic fatty liver disease - MRI accurately quantifies hepatic steatosis in NAFLD. Aliment Pharmacol Ther. 2012;36:22-9.

35. Imajo K, Kessoku T, Honda Y, Tomeno W, Ogawa Y, Mawatari H, et al. Magnetic resonance imaging more accurately classifies steatosis and fibrosis in patients with nonalcoholic fatty liver disease than transient elastography. Gastroenterology. 2016;150:626-37.

36. Yoneda M, Yoneda M, Fujita K, Inamori M, Tamano M, Hiriishi H, et al. Transient elastography in patients with non-alcoholic fatty liver disease (NAFLD). Gut. 2007;56:1330-1.

37. Furuta M, Ekuni D, Yamamoto T, Irie K, Koyama R, Sanbe $T$, et al. Relationship between periodontitis and hepatic abnormalities in young adults. Acta Odontol Scand. 2010;68:27-33.

38. Saito T, Shimazaki Y, Koga T, Tsuzuki M, Ohshima A. Relationship between periodontitis and hepatic condition in Japanese women. J Int Acad Periodontol. 2006:8:89-95.

39. Ahmad A, Furuta M, Shinagawa T, Takeuchi K, Takeshita T, Shimazaki Y, et al. Association of periodontal status with liver abnormalities and metabolic syndrome. J Oral Sci. 2015;57:335-43.

40. Morita T, Yamazaki Y, Fujiharu C, Ishii T, Seto M, Nishinoue N, et al. Serum Yglutamyltransferase level is associated with periodontal disease independent of drinking habits in Japanese adults. Med Sci Monit. 2014;20:2109-16. 
41. Furusho H, Miyauchi M, Hyogo H, Inubushi T, Ao M, Ouhara K, et al. Dental infection of Porphyromonas gingivalis exacerbates high fat diet-induced steatohepatitis in mice. J Gastroenterol. 2013;48:1259-70.

42. Arimatsu K, Yamada H, Miyazawa H, Minagawa T, Nakajima M, Ryder Ml, et al. Oral pathobiont induces systemic inflammation and metabolic changes associated with alteration of gut microbiota. Sci Rep. 2014;4:4828

43. Nakajima M, Arimatsu K, Kato T, Matsuda Y, Minagawa T, Takahashi N, et al. Oral administration of $P$ gingivalis induces dysbiosis of gut microbiota and impaired barrier function leading to dissemination of enterobacteria to the liver. PloS One. 2015;10:e0134234.

44. Matsuda Y, Kato T, Takahashi N, Nakajima M, Arimatsu K, Minagawa T, et al. Ligature-induced periodontitis in mice induces elevated levels of circulating interleukin-6 but shows only weak effects on adipose and liver tissues. J Periodontal Res. 2016;51:639-46.

45. The Helsinki Declaration of the World Medical Association (WMA). Ethical principles of medical research involving human subjects. Pol Merkur Lekarski. 2014;36:298-301.

46. Miyazawa Y. Diagnostic tests approved by Ministry of Health and Welfare (September/October 2011). Rinsho Byori. 2011;59:1055-62.

47. Schulz KF, Altman DG, Moher D, CONSORT Group. CONSORT 2010

Statement: updated guidelines for reporting parallel group randomised trials. Ann Inter Med. 2010;152:726-32.

\section{Publisher's Note}

Springer Nature remains neutral with regard to jurisdictional claims in published maps and institutional affiliations.

Ready to submit your research? Choose BMC and benefit from:

- fast, convenient online submission

- thorough peer review by experienced researchers in your field

- rapid publication on acceptance

- support for research data, including large and complex data types

- gold Open Access which fosters wider collaboration and increased citations

- maximum visibility for your research: over $100 \mathrm{M}$ website views per year

At BMC, research is always in progress.

Learn more biomedcentral.com/submissions 